\title{
THE OPEN MAPPING PRINCIPLE FOR PARTIAL ACTIONS OF POLISH GROUPS
}

\author{
J. GÓMEZ, H. PINEDO, AND C. UZCÁTEGUI
}

\begin{abstract}
We present a extension of the classical open mapping principle and Effros' theorem for Polish group actions to the context of partial group actions.
\end{abstract}

\section{INTRODUCTION}

Let $a: G \times \mathbb{X} \rightarrow \mathbb{X}$ be a continuous action of a Polish group $G$ on a non meager, separable metrizable space $\mathbb{X}$. The open mapping principle states that, if $a$ is transitive, then the evaluation map $g \mapsto a(g, x)$ is open, for each $x \in \mathbb{X}$. The first proof of this result is due to Effros [7], since then several other proofs has been found $[3,10,12,18]$. The original statement of Effros' theorem says that for a Polish space $\mathbb{X}$, the orbit of point $G \cdot x$ is Polish iff $\mathbb{X}$ is homeomorphic to the coset space $G / G_{x}$ (where $G_{x}$ is the stabilizer of $x$ ). This result is fundamental for the classification of orbit equivalence relations [10]. Moreover, motivated by Effros' theorem, the question of which Polish spaces admit a transitive action of a Polish group (and therefore are homeomorphic to a coset space) has been investigated [19]. Effros' results turned out to have an important influence upon the development of the theory of homogeneous continua [4]. Generalization of Effros' theorem are discussed in $[17,20]$.

Given an action $a: G \times \mathbb{Y} \rightarrow \mathbb{Y}$ of a group $G$ over a set $\mathbb{Y}$ and an invariant subset $\mathbb{X}$ of $\mathbb{Y}$ (i.e. $a(g, x) \in \mathbb{X}$, for all $x \in \mathbb{X}$ and $g \in G)$, the restriction of $a$ to $G \times \mathbb{X}$ is an action of $G$ over $\mathbb{X}$. However, if $\mathbb{X}$ is not invariant, we get what is called a partial action on $\mathbb{X}$ : a collection of partial maps $\left\{m_{g}\right\}_{g \in G}$ on $\mathbb{X}$ satisfying $m_{1}=\mathrm{id}_{\mathbb{X}}$ and $m_{g} \circ m_{h} \subseteq m_{g h}$, for all $g, h \in G$. The notion of partial action of a group is a weakening of classical group actions and was introduced by R. Exel in [9] motivated by problems arising from $C^{*}$-algebras, in particular, they have been useful to endow relevant classes of $C^{*}$-algebras with a general structure of a partial crossed product (see for instance $[2,8])$. Recently, partial actions of groups have been considered in many different contexts, [14, p. 89], moreover, they have been an efficient tool to develop a new cohomological theory [6, 21].

In the topological context, partial actions were introduced in $[1,14]$. They consist of a family of homeomorphism between a pair of open subsets of the spaces. A natural question is whether a partial action of a group $G$ can be realized as a restriction of a global action of $G$. This problem was studied by Abadie [1] and independently by J. Kellendonk and M. Lawson [14]. They showed that for any continuous partial action $m$ of a topological group $G$ on a topological space $\mathbb{X}$, there is a topological space $\mathbb{Y}$ and a continuous action $a$ of $G$ on $\mathbb{Y}$ such that $\mathbb{X}$ is a subspace of $\mathbb{Y}$ and $m$ is the restriction of $a$ to $\mathbb{X}$. Such a space $\mathbb{Y}$ is called a globalization of $\mathbb{X}$. They also show that there is a minimal globalization $\mathbb{X}_{G}$ called the enveloping space of $\mathbb{X}$. Partial actions of Polish groups on Polish spaces were studied in $[11,22,23]$. In particular, sufficient conditions for the space $\mathbb{X}_{G}$ to be Polish were found (see Theorem 2.5 below).

Date: April 16, 2018.

1991 Mathematics Subject Classification. 54H15, 54E50, 54E35.

Key words and phrases. Partial action, Polish group, Polish space, open mapping theorem.

The authors thank La Vicerrectoría de Investigación y Extensión de la Universidad Industrial de Santander for the financial support for this work, which is part of the VIE project \# 5761. 
The main purpose of this paper is to show the open mapping principle and Effros' theorem in the setting of partial actions of Polish groups (see Theorems 3.5, 4.2 and 4.5). It is worth to notice that in the case the space $\mathbb{X}_{G}$ is metrizable, the open mapping principle for partial actions can be obtained as a corollary of the corresponding result for global actions (see Remark 3.11).

\section{Preliminaries}

Throughout this work $G$ will be a topological group with identity $1, \mathbb{X}$ a topological space unless it is said otherwise. A Polish space is a topological space which is is separable and completely metrizable, and a Polish group is a topological group whose topology is Polish. We use [10, 13] as a general reference for descriptive set theoretic notions and results.

We recall the definition of a partial action. Let $m: G \times \mathbb{X} \rightarrow \mathbb{X},(g, x) \mapsto m(g, x)=g \cdot x \in \mathbb{X}$, be a partially defined function. As usual, we write $\exists g \cdot x$ to mean that $(g, x)$ is in the domain of $m$. Then $m$ is called a (set theoretic) partial action of $G$ on $\mathbb{X}$, if for all $g, h \in G$ and $x \in \mathbb{X}$ we have:

(PA1) $\exists g \cdot x$ implies $\exists g^{-1} \cdot(g \cdot x)$ and $g^{-1} \cdot(g \cdot x)=x$,

(PA2) $\exists g \cdot(h \cdot x)$ implies $\exists(g h) \cdot x$ and $g \cdot(h \cdot x)=(g h) \cdot x$,

(PA3) $\exists 1 \cdot x$, and $1 \cdot x=x$.

Let $x \in \mathbb{X}$. Then $G^{x} \cdot x=\left\{g \cdot x \mid g \in G^{x}\right\}$ and $G_{x}=\left\{g \in G^{x} \mid g \cdot x=x\right\}$, are orbit and the stabilizer of $x$, respectively. We say that a partial action $m$ is transitive, if given $x, y \in \mathbb{X}$ there exists $g \in G$ such that $x \in \mathbb{X}_{g^{-1}}$ and $g \cdot x=y$. Equivalently, $m$ is transitive, if and only if, $G^{x} \cdot x=\mathbb{X}$, for all $x \in \mathbb{X}$.

The following lemma is clear.

Lemma 2.1. Let $m$ be a partial action of $G$ in $\mathbb{X}$ and $(g, x) \in G * \mathbb{X}$. Then

(i) For $h \in G$, if $g^{-1} h \in G_{x}$ then $h \in G^{x}$.

(ii) The map $r_{g^{-1}}: G \ni h \mapsto h g^{-1} \in G$ is a homeomorphism with $r_{g^{-1}}\left(G^{x}\right)=G^{g \cdot x}$. In particular, if $m$ is transitive and $G^{x}$ is open, then $G^{y}$ is open, for all $y \in \mathbb{X}$.

We consider $G \times \mathbb{X}$ with the product topology. The domain of the partial action is the set $G * \mathbb{X}=\{(g, x) \in G \times \mathbb{X} \mid \exists g \cdot x\}$ endowed with the induced topology. For $g \in G$ and $x \in \mathbb{X}$ write $\mathbb{X}_{g^{-1}}=\{x \in \mathbb{X} \mid \exists g \cdot x\}$, and $G^{x}=\{g \in G \mid \exists g \cdot x\}$. Then we obtain the family of maps $m_{g}: \mathbb{X}_{g^{-1}} \ni x \mapsto g \cdot x \in \mathbb{X}_{g}$, for all $g \in G$. By [24, Lemma 1.2] a partial action $m: G * \mathbb{X} \rightarrow \mathbb{X}$ can equivalently be formulated in terms of a family of bijections $m=\left\{m_{g}: \mathbb{X}_{g^{-1}} \rightarrow \mathbb{X}_{g}\right\}_{g \in G}$ in the following sense.

Proposition 2.2. A partial action $m$ of $G$ on $\mathbb{X}$ is a family $m=\left\{m_{g}: \mathbb{X}_{g^{-1}} \rightarrow \mathbb{X}_{g}\right\}_{g \in G}$, where $\mathbb{X} g \subseteq \mathbb{X}, m_{g}: \mathbb{X}_{g^{-1}} \rightarrow \mathbb{X}_{g}$ is bijective, for all $g \in G$, and such that:

(i) $\mathbb{X}_{1}=\mathbb{X}$ and $m_{1}=\mathrm{id}_{\mathbb{X}}$

(ii) $m_{g}\left(\mathbb{X}_{g^{-1}} \cap \mathbb{X}_{h}\right)=\mathbb{X}_{g} \cap \mathbb{X}_{g h}$;

(iii) $m_{g} m_{h}: \mathbb{X}_{h^{-1}} \cap \mathbb{X}_{h^{-1} g^{-1}} \rightarrow \mathbb{X}_{g} \cap \mathbb{X}_{g h}$, and $m_{g} m_{h}=m_{g h}$ in $\mathbb{X}_{h^{-1}} \cap \mathbb{X}_{g^{-1} h^{-1}}$;

for all $g, h \in G$.

Definition 2.3. A topological partial action of $G$ on $\mathbb{X}$ is a partial action $m=\left\{m_{g}: \mathbb{X}_{g^{-1}} \rightarrow \mathbb{X}_{g}\right\}_{g \in G}$ on the underlying set $\mathbb{X}$, such that each $\mathbb{X}_{g}$ is open in $\mathbb{X}$, and each $m_{g}$ is a homeomorphism. $A$ continuous partial action is a topological partial action $m: G * \mathbb{X} \rightarrow \mathbb{X}$ which is continuous.

Now we recall the definition of the enveloping action in the topological sense. Let $m$ be a topological partial action of $G$ on $\mathbb{X}$. Define the following equivalence relation on $G \times \mathbb{X}$ :

$$
(g, x) R(h, y) \Longleftrightarrow x \in \underset{2}{\mathbb{X}_{g^{-1} h}} \text { and } m_{h^{-1} g}(x)=y,
$$


and denote by $[g, x]$ the equivalence class of the pair $(g, x)$. The enveloping space of $\mathbb{X}$ is the set $\mathbb{X}_{G}=(G \times \mathbb{X}) / R$ endowed with the quotient topology. Let

$$
q: G \times \mathbb{X} \ni(g, x) \rightarrow[g, x] \in \mathbb{X}_{G}
$$

be the quotient map. An action of $G$ on $\mathbb{X}_{G}$ is defined by

$$
\mu: G \times \mathbb{X}_{G} \ni(g,[h, x]) \rightarrow[g h, x] \in \mathbb{X}_{G} .
$$

The map

$$
\iota: \mathbb{X} \ni x \mapsto[1, x] \in \mathbb{X}_{G}
$$

induces a morphism $\iota: m \rightarrow \mu$ in the category of topological partial actions of $G$ (see [1, page 17]). Next theorem collects some facts that will be used in the sequel.

Theorem 2.4. [1, Theorem 1.1] and [14, Theorem 3.9]. Let $m$ be a continuous partial action of $G$ on $\mathbb{X}$. Then

(1) $q$ is continuous and open.

(2) $\mu$ is a continuous action of $G$ on $\mathbb{X}_{G}$.

(3) $\iota: \mathbb{X} \rightarrow \iota(\mathbb{X})$ is a homeomorphism.

(4) If $G * \mathbb{X}$ is open, then $\iota(\mathbb{X})$ is open.

In general, the space $\mathbb{X}_{G}$ is not metrizable. Next result gives sufficient conditions to get the metrizability of $\mathbb{X}_{G}$.

Theorem 2.5. [22, Theorem 4.7] Let $m$ be a continuous partial action of a separable metrizable group $G$ on a separable metrizable space $\mathbb{X}$, then $\mathbb{X}_{G}$ is metrizable under any of the following conditions: $G * \mathbb{X}$ is clopen or $\mathbb{X}$ is locally compact and $R$ is closed in $(G \times \mathbb{X})^{2}$. If in addition, $G$ and $\mathbb{X}$ are Polish, then $\mathbb{X}_{G}$ is Polish.

We finish this section with a result that will be needed later.

Lemma 2.6. Let $m$ be a partial action of a metrizable group $G$ over a space $\mathbb{X}$. Then the stabilizer $G_{x}$ of $x$ respect to $m$ is equal to the stabilizer of $\iota(x)$ respect to $\mu$, and therefore, if $\mathbb{X}_{G}$ is $T_{1}$ then $G_{x}$ is closed.

Proof. Let $g \in G_{x}$, then $\exists g \cdot x$ and $g \cdot x=x$, which implies $[1, g \cdot x]=[1, x]$, but $[1, g \cdot x]=[g, x]$. Thus by $(2.2)$ we have $\mu_{g}([1, x])=[1, x]$ and $g \in G_{\iota(x)}$. Conversely, if $[g, x]=[1, x]$ then $\exists g \cdot x$ and $g \cdot x=x$, that is $g \in G_{x}$. Finally, if $\mathbb{X}_{G}$ is $T_{1}$, then $\{\iota(x)\}$ is closed in $\mathbb{X}_{G}$ and $G_{\iota(x)}=\mu_{\iota(x)}^{-1}(\{\iota(x)\})$ is closed in $G$.

\section{THE OPEN MAPPING THEOREM}

The purpose of this section is to prove that for a transitive and continuous partial action $m$, the map $m^{x}: G^{x} \ni g \mapsto g \cdot x \in \mathbb{X}$ is open, provided that $G$ is Polish and $G^{x}$ is open for all $x$.

As in most proofs of this type of results, we will use Baire category methods. The proof will follow the ideas presented in [18]. We start by recalling some notions we will need. A subset $A$ of a topological space $\mathbb{X}$ is nowhere meager in $\mathbb{X}$, if every non empty relative open subset of $A$ is not meager in $\mathbb{X}$. A subset of $\mathbb{X}$ is analytic if it is a continuous image of a Polish space (notice that we are not assuming for this definition that $\mathbb{X}$ is Polish, not even metrizable).

The second part of the following proposition generalizes [18, Proposition 2.2]. Since we need this result for Hausdorff spaces, we include a sketch of its proof for the sake of completeness.

Proposition 3.1. Let $\mathbb{X}$ be a Hausdorff space and $A \subseteq \mathbb{X}$ a set with the Baire property. If $A$ is dense and nowhere meager, then $A$ is comeager. In particular, if $\mathbb{X}$ is not meager and $A$ and $B$ are analytic, dense and nowhere meager subsets of $\mathbb{X}$, then $A \cap B \neq \emptyset$. 
Proof. The first claim is straightforward. For the second claim, it suffices to show that every analytic set on a Hausdorff space has the Baire property and then use the obvious fact that the intersection of two comeager sets is comeager and hence non empty as $\mathbb{X}$ is not meager. The proof that an analytic subset of a Hausdorff space has the Baire property uses two facts: (i) the classical result saying that the collection of Baire measurable subsets of a topological space is closed under the Suslin operation (see $[15, \S 11 \mathrm{VII}]$ ) and (ii) any analytic set is the result of applying the Suslin operation to a collection of closed sets (here we need that $\mathbb{X}$ is Hausdorff) .

It is a standard fact that in any first countable group there is a basis $\left\{U_{n}\right\}_{n}$ of the identity $1 \in G$ with the following properties:

(a) $U_{n}$ is symmetric $\left(U_{n}=U_{n}^{-1}\right)$, for each $n \in \mathbb{N}$ and $U_{1}=G$;

(b) $U_{n+1} \subseteq U_{n+1}^{2} \subseteq U_{n}$.

We fix such a basis for the rest of the paper.

Given $U \subseteq G, A \subseteq \mathbb{X}, g \in G$ and $x \in \mathbb{X}$, we denote $U^{x}=U \cap G^{x}=\{h \in U: \exists h \cdot x\}$, and $g \cdot A=m_{g}\left(A \cap \mathbb{X}_{g^{-1}}\right)$.

Lemma 3.2. Let $U, F \subseteq G$ and $x \in \mathbb{X}$. Then $\left(\bigcup_{g \in F}\left(g U^{x}\right)\right)^{x} \cdot x=\bigcup_{g \in F}\left(g \cdot U^{x}\right) \cdot x$.

Proof. Indeed, for $y \in\left(\bigcup_{g \in F}\left(g U^{x}\right)\right)^{x} \cdot x$, there is $g \in F$ and $u \in U^{x}$ such that $y=(g u) \cdot x$, then $x \in \mathbb{X}_{(g u)^{-1}} \cap \mathbb{X}_{u^{-1}}$, and by (ii) of Proposition 2.2 we get that $u \cdot x \in \mathbb{X}_{g^{-1}} \cap \mathbb{X}_{u}$, which implies $y=g \cdot(u \cdot x)$, thanks to (PA2), from this we get that $y \in \bigcup_{g \in F}\left(g \cdot U^{x}\right) \cdot x$. The converse is easy.

Lemma 3.3. Let $m$ be a continuous partial action of $G$ on $\mathbb{X}$. Let $n \in \mathbb{N}$ and $x \in \mathbb{X}$ such that $G^{x}$ is open. Then for every open subset $V$ of $\mathbb{X}$ and $z \in V \cap U_{n}^{x} \cdot x$, there is $m \in \mathbb{N}$ such that $U_{m}^{z} \cdot z \subseteq V \cap U_{n}^{x} \cdot x$.

Proof. First of all notice that $U_{n}^{x}=U_{n} \cap G^{x}$ is an open subset of $G$ containing the identity. Since $z \in V \cap U_{n}^{x} \cdot x$, then $z=m^{x}(h)$ for some $h \in U_{n}^{x}$. Let $E=\left(m^{x}\right)^{-1}(V)$, then $E$ is an open subset of $G$. But $1 \in E h^{-1} \cap U_{n}^{x} h^{-1}$, thus there is $m \in \mathbb{N}$ such that $U_{m} \subseteq E h^{-1} \cap U_{n}^{x} h^{-1}$. Now we check that $U_{m}^{z} \cdot z \subseteq V \cap U_{n}^{x} \cdot x$. Let $p \in U_{m}^{z} \cdot z$ and $g \in U_{m}^{z}$ such that $p=g \cdot z$. Since $g \in U_{m}^{z} \subseteq E h^{-1} \cap U_{n}^{x} h^{-1}$, then $g h \in E \cap U_{n}^{x}$ and

$$
p=g \cdot z=g \cdot(h \cdot x) \stackrel{(\mathrm{PA} 2)}{=}(g h) \cdot x=m^{x}(g h) \in m^{x}\left(E \cap U_{n}^{x}\right) .
$$

Therefore $p \in V \cap U_{n}^{x} \cdot x$, as desired.

Next lemma is the crucial step for the proof of our main result.

Lemma 3.4. Let $G$ be a Polish group and $m$ a continuous transitive partial action of $G$ on a non meager Hausdorff space $\mathbb{X}$ such that $G^{x}$ is open for all $x \in \mathbb{X}$. Then the following holds for all $x \in \mathbb{X}$.

(i) $U_{n}^{x} \cdot x$ is not meager for every $n \in \mathbb{N}$.

(ii) $U_{n}^{x} \cdot x$ is nowhere meager for every $n \in \mathbb{N}$. In particular, $\mathbb{X}$ is Baire.

(iii) int $\left(\overline{U_{n}^{x} \cdot x}\right)$ is dense in $\overline{U_{n}^{x} \cdot x}$ and $x \in \operatorname{int}\left(\overline{U_{n}^{x} \cdot x}\right)$ for every $n \in \mathbb{N}$.

(iv) $\operatorname{int}\left(\overline{U_{n+1}^{x} \cdot x}\right) \subseteq U_{n}^{x} \cdot x$.

(v) $x \in \operatorname{int}\left(U_{n}^{x} \cdot x\right)$ for every $n \in \mathbb{N}$.

(vi) $U_{n}^{x} \cdot x$ is an open neighborhood of $x$ for every $n \in \mathbb{N}$.

Proof. (i) Suppose that $U_{n}^{x} \cdot x$ is meager. Notice that $\left\{g U_{n}^{x}\right\}_{g \in G}$ is a open cover of $G$. Also, since $G$ is metrizable and separable, it is Lindelöf, then there is a countable set $F$ such that $G=\bigcup_{g \in F} g U_{n}^{x}$. 
By Lemma 3.2,

$$
\mathbb{X}=G^{x} \cdot x=\bigcup_{g \in F} g \cdot\left(U_{n}^{x} \cdot x\right) .
$$

For each $g \in F$, the set $\left(U_{n}^{x} \cdot x\right) \cap \mathbb{X}_{g^{-1}}$ is meager in $\mathbb{X}_{g^{-1}}$ (as $\mathbb{X}_{g^{-1}}$ is open), thus $g \cdot\left(U_{n}^{x} \cdot x\right)=$ $m_{g}\left(U_{n}^{x} \cdot x \cap \mathbb{X}_{g^{-1}}\right)$ is meager in $\mathbb{X}_{g}$ and hence in $\mathbb{X}$. Since $F$ is countable, $\mathbb{X}$ is meager, which is a contradiction.

(ii) We shall prove that every non empty relative open subset of $U_{n}^{x} \cdot x$ is not meager. Let $V$ be an open subset of $\mathbb{X}$ and $z \in V \cap U_{n}^{x} \cdot x$, by Lemma 3.3, there is $m \in \mathbb{N}$ such that $U_{m}^{z} \cdot z \subseteq V \cap U_{n}^{x} \cdot x$. By (i), $U_{m}^{z} \cdot z$ is not meager. Therefore $V \cap U_{n}^{x} \cdot x$ is not meager. This finishes the proof that $U_{n}^{x} \cdot x$ is nowhere meager. Since $U_{1}=G$ and $\mathbb{X}=G^{x} \cdot x$, then $\mathbb{X}$ is nowhere meager, i.e. it is Baire.

(iii) Let $V$ be a open subset of $\mathbb{X}$ such that $V \cap \overline{U_{n}^{x} \cdot x} \neq \emptyset$. By (ii), the set $V \cap U_{n}^{x} \cdot x$ is not meager, in particular, int $\left(\overline{V \cap U_{n}^{x} \cdot x}\right) \neq \emptyset$. From this it follows that int $\left(\overline{U_{n}^{x} \cdot x}\right)$ is dense in $\overline{U_{n}^{x} \cdot x}$.

To see the second claim, let $V$ be a non empty open subset of $\mathbb{X}$ such that $V \subseteq \overline{U_{n+1}^{x} \cdot x}$. Then there is $h \in U_{n+1}^{x}$ such that $h \cdot x \in V$. Since $U_{n+1} \subseteq U_{n+1}^{2} \subseteq U_{n}$ and $m_{h^{-1}}$ is continuous, then

$$
\begin{aligned}
x \in h^{-1} \cdot\left(V \cap \mathbb{X}_{h}\right) & \subseteq h^{-1} \cdot\left(\overline{U_{n+1}^{x} \cdot x} \cap \mathbb{X}_{h}\right) \\
& \subseteq \overline{h^{-1} \cdot\left(\left(U_{n+1}^{x} \cdot x\right) \cap \mathbb{X}_{h}\right)} \\
& =\overline{\left\{\left(h^{-1} g\right) \cdot x: g \in U_{n+1}^{x}\right\}} \\
& \subseteq \overline{U_{n}^{x} \cdot x} .
\end{aligned}
$$

Finally, since $m_{h^{-1}}$ is a homeomorphism and $\mathbb{X}_{h}$ and $\mathbb{X}_{h^{-1}}$ are open, then $h^{-1} \cdot\left(V \cap \mathbb{X}_{h}\right)$ is an open subset of $\mathbb{X}$ containing $x$; thus $x \in \operatorname{int}\left(\overline{U_{n}^{x} \cdot x}\right)$.

(iv) Let $z \in V=\operatorname{int}\left(\overline{U_{n+1}^{x} \cdot x}\right), W=\operatorname{int}\left(\overline{U_{n+1}^{z} \cdot z}\right)$ and $E=V \cap W$. Thus $E$ is a open neighborhood of $z$ and it is not meager as $\mathbb{X}$ is Baire (by (ii)). Clearly $U_{n+1}^{x} \cdot x \cap E$ and $U_{n+1}^{z} \cdot z \cap E$ are dense in $E$ and by (ii) those sets are also nowhere meager in $E$. Since $G$ is Polish and each $U_{m}^{y}$ is open, then $U_{m}^{y}$ is also Polish for every $y$ and $m$. Thus $U_{n+1}^{x} \cdot x$ and $U_{n+1}^{z} \cdot z$ are analytic. In summary, $U_{n+1}^{x} \cdot x \cap E$ and $U_{n+1}^{z} \cdot z \cap E$ are analytic, dense and nowhere meager subsets of $E$ (as a subspace of $\mathbb{X})$, then by Proposition 3.1, there exists

$$
y \in\left(U_{n+1}^{x} \cdot x \cap E\right) \cap\left(U_{n+1}^{z} \cdot z \cap E\right) .
$$

Hence, there are $g \in U_{n+1}^{x}$ and $h \in U_{n+1}^{z}$ such that $g \cdot x=y=h \cdot z$. Let $f=h^{-1} g$, then $f \cdot x=z$. Note that $f \in U_{n+1} U_{n+1} \subseteq U_{n}$ and $f \in G^{x}$, then $f \in U_{n}^{x}$. Hence $z \in U_{n}^{x} \cdot x$.

(v) By (iv), int $\left(\overline{U_{n+1}^{x} \cdot x}\right) \subseteq U_{n}^{x} \cdot x$ and thus int $\left(\overline{U_{n+1}^{x} \cdot x}\right) \subseteq \operatorname{int}\left(U_{n}^{x} \cdot x\right)$. From (iii) we have that $x \in \operatorname{int}\left(\overline{U_{n+1}^{x} \cdot x}\right)$. Therefore $x \in \operatorname{int}\left(U_{n}^{x} \cdot x\right)$.

(vi) Let $z \in U_{n} \cdot x$. By Lemma 3.3 there exists $m \in \mathbb{N}$ such that $U_{m}^{z} \cdot z \subseteq U_{n}^{x} \cdot x$ and by (v), $z \in \operatorname{int}\left(U_{m}^{z} \cdot z\right)$. Thus $z \in \operatorname{int}\left(U_{m}^{z} \cdot z\right) \subseteq U_{n}^{x} \cdot x$. Hence $U_{n}^{x} \cdot x$ is open in $\mathbb{X}$.

Now we are ready to give the proof of the open mapping theorem for partial actions.

Theorem 3.5. Let $G$ be a Polish group and $m$ a continuous transitive partial action of $G$ in a not meager Hausdorff space $\mathbb{X}$. Suppose $G^{y}$ is open in $G$ for some $y \in \mathbb{X}$. Then the map $m^{x}: G^{x} \ni$ $g \mapsto g \cdot x \in \mathbb{X}$ is open for every $x \in \mathbb{X}$.

Proof. By (ii) of Lemma 2.1 we assume that $G^{x}$ is open for all $x \in \mathbb{X}$. Let $U^{x}$ be an open non empty subset of $G^{x}$, where $U$ is an open subset of $G$. We check that $U^{x} \cdot x$ is an open subset of $G^{x} \cdot x$. Indeed, given $z \in U^{x} \cdot x$, then $z=g \cdot x$, for some $g \in U^{x}$. As $U$ is open, there are $O_{1}$ and $O_{g}$ open neighborhoods of 1 and $g$, respectively, such that $O_{1} O_{g} \subseteq U$ and thus $O_{1}^{z} g \subseteq U^{x}$. Since $\left\{U_{n}\right\}_{n}$ is a basis of neighborhoods of 1 , there exists $n \in \mathbb{N}$ such that $U_{n}^{z} \subseteq O_{1}^{z}$. Note that $z \in U_{n}^{z} \cdot z$ and $U_{n}^{z} \cdot z \subseteq O_{1}^{z} \cdot z=O_{1}^{z} \cdot(g \cdot x)=\left(O_{1}^{z} g\right) \cdot x \subseteq U^{x} \cdot x$. Thus, by Lemma 3.4 (vi), the set $U_{n}^{z} \cdot z$ is open and hence $U^{x} \cdot x$ is also open. 
Example 3.6. Möbius transfromations [5, p. 175] The group $G=\operatorname{SL}(2, \mathbb{R})$ acts partially on $\mathbb{R}$ by setting

$$
g \cdot x=\frac{a x+b}{c x+d}, \quad \text { where } \quad g=\left(\begin{array}{ll}
a & b \\
c & d
\end{array}\right) \in G .
$$

Notice that for all $g \in G$ the set $\mathbb{X}_{g}=\{x \in \mathbb{R} \mid c x+d \neq 0\}$ is open and the partial action is continuous. For $x \in \mathbb{R}$ let $t_{x}=\left(\begin{array}{ll}1 & x \\ 0 & 1\end{array}\right)$, then for $y \in \mathbb{R}$ one has that $t_{y-x} \cdot x=y$. Moreover, since

$$
G^{0}=\left\{\left(\begin{array}{cc}
a & b \\
c & d
\end{array}\right) \in G: d \neq 0\right\}
$$

is open, then by (ii) of Lemma 2.1 we have a transitive partial action for which $G^{x}$ is open, for all $x \in \mathbb{R}$. By Theorem 3.5, the map $m^{x}: G^{x} \ni g \mapsto g \cdot x \in \mathbb{R}$ is open, for every $x \in \mathbb{R}$.

3.1. On the enveloping space of a transitive partial action. In this section we use the open mapping principle to get an improvement of Theorem 2.5 for transitive partial actions. We recall a result that gives a (set theoretic) relation between the enveloping space $\mathbb{X}_{G}$ and the quotient $G / G_{x}$, for $x \in \mathbb{X}$.

Theorem 3.7. [5, Proposition 2.4, Theorem 2.6] Let $m$ be a transitive partial action of a group $G$ over $\mathbb{X}$. Then the enveloping action $\mu$ of $G$ over $\mathbb{X}_{G}$ is transitive and equivalent to the left coset action of $G$ over $G / G_{x}$. More precisely, the map

$$
\phi: G / G_{x} \ni g G_{x} \rightarrow[g, x] \in \mathbb{X}_{G}
$$

is a bijection such that $\phi\left(h g G_{x}\right)=\mu_{h} \phi\left(g G_{x}\right)$, for any $h \in G$.

The following result is straightforward.

Lemma 3.8. Let $G$ be a Polish group and $m$ a transitive continuous partial action of $G$ on a space $\mathbb{X}$. Then the map $\phi$ defined in (3.1) is continuous.

Now we show that, for transitive actions, $\mathbb{X}_{G}$ is Polish when it is Hausdorff.

Theorem 3.9. Let $G$ be a Polish group and $m$ a continuous transitive partial action of $G$ on a not meager space $\mathbb{X}$ such that $G * \mathbb{X}$ is open. The following statements are equivalent.

(1) $\mathbb{X}_{G}$ is Hausdorff.

(2) $\mathbb{X}_{G}$ is $T_{1}$ and is homeomorphic to $G / G_{x}$, for any $x \in \mathbb{X}$.

(3) $\mathbb{X}_{G}$ is Polish.

Proof. (1) $\Rightarrow(2)$. Suppose that $\mathbb{X}_{G}$ is Hausdorff. Since $\iota$ is an embedding (see Theorem 2.4), then $\mathbb{X}$ is Hausdorff. Let $x \in \mathbb{X}$. We will show that $\phi$ is an homeomorphism. By Lemma 3.8, we only need to show that $\phi$ is open. First we verify that we can apply the open mapping Theorems to the enveloping total action $\mu$ of $G$ over $\mathbb{X}_{G}$. In fact, $\mathbb{X}_{G}$ is not meager in itself, as the map $q$ defined in (2.1) is continuous and open and $\mathbb{X}$ and $G$ are not meager. By Theorems 3.7 and 2.4, $\mu$ is a continuous transitive action. Thus, by Theorem 3.5, the map $\mu^{[1, x]}: G \rightarrow \mathbb{X}_{G}$ is open. Now we show that $\phi$ is open. Let $\pi: G \rightarrow G / G_{x}$ be the quotient map. It suffices to show that if $O \subseteq G$ is open, then $\phi(\pi(O))$ is open in $\mathbb{X}_{G}$. In fact, since $\phi \circ \pi=\mu^{[1, x]}$ we are done.

$(2) \Rightarrow(3)$. It is a classical result that if $H$ is a closed subgroup of Polish group $G$, then $G / H$ is also Polish (see [10, Theorem 2.2.10]). But by Lemma 2.6 we know that $G_{x}$ is closed.

$(3) \Rightarrow(1)$ This part is obvious.

Corollary 3.10. Let $G$ be a Polish group and $m$ a continuous transitive partial action of $G$ on a not meager space $\mathbb{X}$ such that $G * \mathbb{X}$ is open. If $\mathbb{X}_{G}$ is Hausdorff, then $\mathbb{X}$ is Polish. 
Proof. By Theorem 2.4, $\iota(\mathbb{X})$ is open and $\iota$ is an embedding, therefore $\mathbb{X}$ is Polish.

Remark 3.11. Suppose $\mathbb{X}_{G}$ is metrizable, then the argument used in the proof of $(1) \Rightarrow(2)$ in Theorem 3.9 shows that the open mapping theorem 3.5 follows from the corresponding theorem for global actions. To see this, observe that $\iota \circ m^{x}=\mu^{[1, x]} \uparrow G^{x}$, and thus $\iota \circ m^{x}$ is an open map. Since $\iota$ is a continuous injection (see Theorem 2.4), then $m^{x}$ is open.

\section{On the Effros' theOrEM}

In this section we extend Effros' result [7, Theorem 2.1] to the context of partial actions.

Definition 4.1. Let $m$ be a partial action on $\mathbb{X}$. The orbit equivalence relation $E_{G}^{p}$ on $\mathbb{X}$ is defined by

for some $g \in G$.

$$
x E_{G}^{p} y \Longleftrightarrow \exists g \cdot x \text { and } g \cdot x=y,
$$

The set of equivalence classes $\mathbb{X} / E_{G}^{p}$ is endowed with the quotient topology. By [22, Lemma $3.2]$ the quotient map $\mathbb{X} \ni x \mapsto[x] \in \mathbb{X} / E_{G}^{p}$ is continuous and open, from this follows that if $\mathbb{X}$ is second-countable, so is $\mathbb{X} / E_{G}^{p}$.

Theorem 4.2. Let $G$ be a Polish group and $m$ be a continuous partial action of $G$ on the Polish space $\mathbb{X}$. Then the following assertions are equivalent.

(1) $E_{G}^{p}$ is $G_{\delta}$.

(2) $G^{x} \cdot x$ is $G_{\delta}$ in $\mathbb{X}$, for every $x \in \mathbb{X}$.

(3) $\mathbb{X} / E_{G}^{p}$ is $T_{0}$. That is, $\overline{\{x\}} \neq \overline{\{y\}}$ for any $x, y \in \mathbb{X} / E_{G}^{p}$ with $x \neq y$.

Proof. It is shown exactly as in the case of a global action (see for instance [10, Theorem 3.4.4]). Only recall that $\mathbb{X} / E_{G}^{p}$ is second countable.

Proposition 4.3. Let $G$ be a Polish group, $\mathbb{X}$ a metric space and $m$ a topological partial action of $G$ on $\mathbb{X}$. Then for a fixed $x \in \mathbb{X}$ the following assertions hold.

(1) The quotient map $\pi_{p}: G^{x} \ni g \rightarrow g G_{x} \in G^{x} / G_{x}$ is continuous and open.

(2) The map

$$
\hat{\iota}: G^{x} / G_{x} \ni g G_{x} \rightarrow g G_{\iota(x)} \in G / G_{\iota(x)}
$$

is a topological embedding.

(3) If $G^{x}$ is $G_{\delta}$ and $G_{x}$ is closed in $G$, then $G^{x} / G_{x}$ is a Polish space.

Proof. (1) Let $V$ be an open subset of $G^{x}$, and take $W \subseteq G$ open such that $V=W \cap G^{x}$. Notice that $V g=W g \cap G^{x} g$ and $G^{x} g=G^{x}$, for all $g \in G_{x}$. Then $\pi_{p}{ }^{-1} \pi_{p}[V]=V G_{x}=\bigcup_{g \in G_{x}} V g=W G_{x} \cap G^{x}$ is open in $G^{x}$.

(2) It is clear that $\hat{\iota}$ is well defined and injective. Moreover, $\hat{\iota} \circ \pi_{p}=\pi \uparrow G^{x}$, where $\pi$ is the canonical projection $G \rightarrow G / G_{\iota(x)}$, then $\hat{\imath} \circ \pi_{p}$ is continuous, which implies that $\hat{\imath}$ is continuous. Let $Y=\hat{\imath}\left[G^{x} / G_{x}\right]$. We will show that $\hat{\iota}: G^{x} / G_{x} \rightarrow Y$ is open. Let $U \subseteq G$ open we prove that

$$
\left\{g G_{\iota(x)}: g \in U \cap G^{x}\right\}=\pi[U] \cap Y .
$$

It is clear that $\subseteq$ holds. For the other inclusion, let $h \in U$ such that $\pi(h) \in Y$. Let $g \in G^{x}$ such that $h G_{\iota(x)}=g G_{\iota(x)}$. Since $G_{x}=G_{\iota(x)}$, then $g^{-1} h \in G_{x}$ and by (i) of Lemma 2.1 we have that $h \in G^{x}$.

(3) Since $G_{x}=G_{\iota(x)}$ is a closed subgroup of $G$ the space $G / G_{\iota(x)}$ is Polish. By $(2), G^{x} / G_{x} \simeq$ $\hat{\iota}\left[G^{x} / G_{x}\right] \subseteq G / G_{\iota(x)}$, thus $G^{x} / G_{x}$ is metrizable. Finally, by (1), $G^{x} / G_{x}$ is the continuous open image of the Polish space $G^{x}$ (as it is a $G_{\delta}$ subset of a Polish space), therefore it is Polish by Sierpinski's theorem (see [10, Theorem 2.2.9]). 
Now we show that the orbits are Borel, this is a generalization of a well known theorem about Polish group actions (see [10, Proposition 3.1.10]).

Corollary 4.4. Let $G$ be a Polish group, $m$ a continuous partial action of $G$ on the Polish space $\mathbb{X}$ and $x \in \mathbb{X}$. If $G^{x}$ is $G_{\delta}$ and $G_{x}$ is closed in $G$, then $G^{x} \cdot x$ is Borel.

Proof. By Lemma $4.3, G^{x} \cdot x$ is the continuous and injective image of the Polish space $G^{x} / G_{x}$, then it is Borel by Lusin-Souslin's Theorem (see [13, Theorem 15.1]).

Theorem 4.5. Let $G$ be a Polish group and $m$ be a continuous partial action of $G$ on the Polish space $\mathbb{X}$. Let $x \in \mathbb{X}$ such that $G^{x}$ is $G_{\delta}$ and $G_{x}$ is closed. Consider the following assertions.

(1) The map

is a homeomorphism.

$$
\phi: G^{x} / G_{x} \ni g G_{x} \rightarrow g \cdot x \in G^{x} \cdot x
$$

(2) $G^{x} \cdot x$ is $G_{\delta}$.

(3) $G^{x} \cdot x$ is not meager in its relative topology.

Then $(1) \Rightarrow(2) \Rightarrow(3)$. Moreover, if $G^{x}$ is open, then all the assertions are equivalent.

Proof. Fix $x \in \mathbb{X}$. Since $G^{x}$ is $G_{\delta}$ and $G_{x}$ is closed, then by (3) of Proposition 4.3 we have (1) $\Rightarrow(2)$. It is clear that $(2) \Rightarrow(3)$. Now suppose that $G^{x}$ is open. It is clear that $\phi$ is a continuous bijection, so to see that $(3) \Rightarrow(1)$ it suffices to show that $\phi$ is open. Let $\mathbb{Y}=G^{x} \cdot x$ and $\bar{m}: G * \mathbb{Y} \rightarrow \mathbb{Y}$ the restriction of $m$. Then $\bar{m}$ is a continuous transitive partial action with $\bar{m}^{x}=m^{x}$. Since $m^{x}=\phi \circ \pi_{p}$, the result follows from Proposition 4.3 (1) and Theorem 3.5.

The following result is a generalization of the fact commented in the introduction. If a Polish space $\mathbb{X}$ admits a transitive action of a Polish group $G$, then $\mathbb{X}$ is homeomorphic to the coset space $G / G_{x}$ for any $x \in \mathbb{X}$.

Corollary 4.6. Let $G$ be a Polish group and $m$ a continuous transitive partial action of $G$ on a not meager Hausdorff space $\mathbb{X}$ such that $G * \mathbb{X}$ is open and $G_{x}$ is closed for some $x \in \mathbb{X}$. Then $\mathbb{X}$ is homeomorphic to the coset space $G^{x} / G_{x}$.

Proof. By Corollary 3.10 the space $\mathbb{X}$ is Polish, then the results follows by Theorem 4.5.

Now we present an example to illustrate Theorems 4.2 and 4.5.

Example 4.7. The flow of a differentiable vector field [1, Example 1.2]. Consider a smooth vector field $V: \mathbb{X} \rightarrow T \mathbb{X}$ on a manifold $\mathbb{X}$ such that $\mathbb{X}$ is a Polish space. For $x \in \mathbb{X}$, let $\gamma_{x}$ be the corresponding integral curve through $x$ defined on its maximal interval $\left(a_{x}, b_{x}\right)$, that is, $\gamma_{x}(0)=x$ and $\gamma_{x}^{\prime}(t)=V\left(\gamma_{x}(t)\right)$ for all $t \in\left(a_{x}, b_{x}\right)$. For $t \in \mathbb{R}$, let $\mathbb{X}_{-t}=\left\{x \in \mathbb{X} \mid t \in\left(a_{x}, b_{x}\right)\right\}$ and set $m_{t}: \mathbb{X}_{-t} \ni x \rightarrow \gamma_{x}(t) \in \mathbb{X}_{t}$. Then the family $m=\left\{m_{t}: \mathbb{X}_{-t} \rightarrow \mathbb{X}_{t}\right\}_{t \in \mathbb{R}}$ defines a continuous partial action of the additive group $G=\mathbb{R}$ on $\mathbb{X}$. Maximal integral curves are either constant, injective or periodic (see [16, Exercise 9-1]). Then for each $x \in \mathbb{X}$, we have that

$$
G_{x}=\left\{t \in\left(a_{x}, b_{x}\right): \gamma_{x}(t)=x\right\}
$$

is a closed set. Additionally, the image of $\gamma_{x}$ is diffeomorphic to $\mathbb{R}, S^{1}$, or $\mathbb{R}^{0}$, thus $G^{x} \cdot x=$ $\gamma_{x}\left(\left(a_{x}, b_{x}\right)\right)$ is Polish (see [16, Exersice 9-1 (c)]).

The following example shows that the condition $G^{x}$ to be Polish in Theorem 4.5 is necessary.

Example 4.8. Consider the continuous partial action of $G=\mathbb{R}$ on itself given by $\mathbb{X}_{g}=\emptyset$ if $g \in \mathbb{R} \backslash \mathbb{Q}, \mathbb{X}_{g}=\mathbb{R}$ if $g \in \mathbb{Q}$, and $m_{g}: \mathbb{X}_{-g} \ni a \rightarrow g+a \in \mathbb{X}_{g}$, for $g \in \mathbb{Q}$. Then for any $x \in \mathbb{Q}$ one has that $G^{x}=\mathbb{Q}$ which is not a Polish subset of $\mathbb{R}$. Moreover $G_{x}=\{0\}$ is closed in $\mathbb{R}$ and the map

$$
G^{x} / G_{x} \ni[r] \mapsto r+x \in G^{x} \cdot x
$$

is a homeomorphism. But $G^{x} \cdot x=\mathbb{Q}$ is meager in itself. 


\section{REFERENCES}

[1] F. Abadie, Enveloping actions and Takai duality for partial actions. Journal of Func. Anal. (2003) 197: 14-67.

[2] F. Abadie, L. Martínez Pérez, On the amenability of partial and enveloping actions, Proc. Amer. Math. Soc. (2009) 137: 689-693.

[3] F. D. Ancel, An alternative proof and applications of a theorem of E. G. Effros, Michigan Math. J. 34 (1987) 3955.

[4] J. J. Charatonik and T. Maćkoviack, Around Effros' Theorem, Trans. Am. Math. Soc. 298 (1986), 579-602.

[5] K. Choi, Y. Lim, Transitive partial actions of groups, Period. Math. Hung. 56, (2008), No. 2, 169-181.

[6] M. Dokuchaev, M. Khrypchenko, Partial cohomology of groups, J. Algebra, 427 (2015), 142-182.

[7] E. G. Effros, Transformations groups and $C^{\star}$-algebras. Annals of Mathematics, (1965) 81, 38-55.

[8] R. Exel, F. Vieira, Actions of inverse semigroups arising from partial actions of groups, J. Math. Anal. Appl. (2010) 363: 86-96.

[9] R. Exel, Partial actions of groups and actions of inverse semigroups, Proc. Am. Math. Soc. 126 (1998), (12), 3481-3494.

[10] Su Gao, Invariant Descriptive Set Theory, Chapmann $\&$ Hall (2009).

[11] J. Gómez , H. Pinedo and C. Uzcátegui , On the continuity of partial actions of Polish groups on metric spaces. arxiv.org/pdf/1708.09474.

[12] A. Hohti, Another alternative proof of Effros theorem, Top. Proc. (1987) 12 295-298.

[13] A. Kechris, Classical Descriptive Set Theory. Springer-Verlag (1995).

[14] J. Kellendonk and M. V. Lawson, Partial actions of groups. Internat. J. Algebra Comput. (2004) 14, 87-114.

[15] K. Kuratowski, Topology, Vol I, Academic Press, New York, N. Y., 1968.

[16] J. Lee, Introduction to Smooth Manifolds, 2nd ed., Springer-Verlag, New York, 2012.

[17] M. Lupini, Polish groupoids and functorial complexity. arxiv.org/abs/1407.6671v2.

[18] J. van Mill, A Note on Effros' Theorem, The Amer. Math. Monthly. (2004) 111 (9), 801-806.

[19] J. van Mill, Homogeneous spaces and transitive actions by Polish groups. Israel J. Math. (2008) 165, $133-159$.

[20] A. J. Ostaszewski, Effros, Baire, Steinhaus and non-separability. Topology Appl., 195, 265-274, 2015.

[21] H. Pinedo, Partial projective representations and the partial Schur multiplier: a survey. Bol. Mat. (2015) (22) 2 $167-175$.

[22] H. Pinedo and C. Uzcátegui, Polish globalization of Polish group partial actions. Math. Log. Quart. To appear.

[23] H. Pinedo and C. Uzcátegui, Borel globalization of partial actions of Polish groups. arxiv.org/pdf/1702.02611.

[24] J.C. Quigg, I. Raeburn, Characterizations of crossed products by partial actions. J.Operator Theory (1997) 37: 311-340.

Escuela de Matemáticas, Universidad Industrial de Santander, Cra. 27 Calle 9 UiS Edificio 45, Bucaramanga, Colombia

E-mail address: jorge.gomez14@correo.uis.edu.co

Escuela de Matemáticas, Universidad Industrial de Santander, Cra. 27 Calle 9 UiS Edificio 45, Buchramanga, Colombia

E-mail address: hpinedot@uis.edu.co

Escuela de Matemáticas, Universidad Industrial de Santander, Cra. 27 Calle 9 Uis Edificio 45, Buchramanga, Colombia

E-mail address: cuzcatea@uis.edu.co 